\title{
Unreliable Batch Arrival Retrial Queue with Negative Customers, Multi-Types of Heterogeneous Service, Setup Time and Reserved Time
}

\author{
K. Kirupa \\ Research Scholar \\ Department of Mathematics \\ Avinashilingam University \\ Coimbatore
}

\author{
K. Udaya Chandrika, $\mathrm{PhD}$ \\ Professor and Head \\ Department of Mathematics \\ Avinashilingam University \\ Coimbatore
}

\begin{abstract}
Batch arrival retrial queue with positive and negative customers is considered. Server provides M types of service. Positive customers arrive in batches according to Poisson process. If the server is idle upon the arrival of a batch, one of the customers in the batch receives any one the types immediately and others join the orbit. The server is subject to two different modes of failure. Mode 1 failure occurs due to the arrival of negative customer and Mode 2 due to random breakdown of the server. In both cases, repair starts after some random amount of time. The server failed under mode 2 continues the interrupted service or waits for the same customer after the repair completion. Generating function technique is employed to obtain joint distributions of the server state and orbit length. Expected system size, expected orbit size, availability of the server and failure frequency of the server are derived. Stochastic decomposition law is also verified.
\end{abstract}

\section{Keywords}

Retrial queue - negative customers - multi-types of heterogeneous service - setup time - reserved time.

\section{INTRODUCTION}

Retrial queueing system are characterized by the fact that an arriving customer who finds the server occupied is obliged to join a group of blocked customers and reapply after random intervals of time to obtain the service. Two extensive survey articles in retrial queue are due to Yang and Templeton [10] and Falin [3].

Queues with server subject to breakdown and repairs are often encountered in many practical applications. Aissani [1] and Kulkarni and Choi [6] considered retrial queueing systems with server breakdowns and repairs. Prakash Rani et al. [8] analysed retrial queueing models with server breakdown and delayed repair. Choudhury and $\mathrm{Ke}$ [2] considered a batch arrival retrial queue with general retrial time, Bernoulli vacation, unreliable server and delayed repair.

Queue with negative arrivals called G-queue was first introduced by Gelenbe [4] with a view to modeling neural networks. Wang and Zhang [9] considered a discrete time retrial queue with negative arrivals. Peng et al. [7] suggested an $\mathrm{M} / \mathrm{G} / 1$ retrial G-queue with pre-emptive resume priority and collisions under linear retrial policy. In this paper, the article by Kirupa and Udaya Chandrika[5] is analyzed by including multi-types of heterogeneous service and reserved time.

\section{MODEL DESCRIPTION}

Single server queueing system with two types of arrival positive and negative is considered. Positive customers arrive according to Poisson process with rate $\lambda^{+}$in groups of random size having distribution function $\mathrm{P}(\mathrm{Y}=\mathrm{k})=\mathrm{C}_{\mathrm{k}}, \mathrm{k} \geq 1$, the generating function $\mathrm{C}(\mathrm{z})$ and first two moments $\mathrm{m}_{1}$ and $\mathrm{m}_{2}$. The server provides $\mathrm{M}$ types of service. Customers opt type $\mathrm{i}$ service with probability $\mathrm{p}_{\mathrm{i}}(1 \leq \mathrm{i} \leq \mathrm{M})$. If the arriving batch of positive customers finds the server free, then one of the arrivals in the batch receives service immediately and others join the orbit. Otherwise the arriving batch joins the retrial queue. The retrial time is generally distributed with distribution function $\mathrm{A}(\mathrm{x})$, density function $\mathrm{a}(\mathrm{x})$, Laplace Stieltje's transform $\mathrm{A}^{*}(\bullet)$ and conditional completion rate $\eta(x)=a(x) /[1-A(x)]$.

The service time of type $i(i=1,2, \ldots, M)$ follows a general distribution with distribution function $\mathrm{B}_{\mathrm{i}}(\mathrm{x})$, density function $\mathrm{b}_{\mathrm{i}}(\mathrm{x})$, Laplace Stieltje's transform $\mathrm{B}_{\mathrm{i}}{ }^{*}(\bullet), \mathrm{n}^{\text {th }}$ factorial moments $\mu_{i, n}$ and conditional completion rate $\mu_{\mathrm{i}}(\mathrm{x})=\mathrm{b}_{\mathrm{i}}(\mathrm{x}) /[1$ $\mathrm{B}_{\mathrm{i}}(\mathrm{x})$ ]. There are two different modes of server breakdown, say mode 1 and mode 2 . Mode 1 is due to negative arrival which removes the positive customer being in service from the system and makes the server down. Mode 2 is random failure of the server. The lifetime of the server providing type i service is exponentially distributed with rate $\alpha_{i}(i=1,2, \ldots, M)$. Once the system fails, the repair starts after a random amount of time. This time is referred as setup time. The setup times of the server failed under mode $l(l=1,2)$ are generally distributed with distribution function $\mathrm{S}_{\mathrm{i}}^{(l)}(\mathrm{x})$, density function $\mathrm{s}_{\mathrm{i}}^{(l)}(\mathrm{x})$, Laplace Stieltje's transform $\mathrm{S}_{\mathrm{i}}^{(l) *}(\bullet), \quad \mathrm{n}^{\text {th }}$ factorial moments $\phi_{\mathrm{i}, \mathrm{n}}^{(l)}$ and conditional completion rate $\phi_{\mathrm{i}}^{(l)}(\mathrm{x})=\mathrm{s}_{\mathrm{i}}^{(l)}(\mathrm{x}) /\left[1-\mathrm{S}_{\mathrm{i}}^{(l)}(\mathrm{x})\right]$.

The repair times of mode $l$ breakdown are generally distributed with distribution function $\mathrm{R}_{\mathrm{i}}^{(l)}(\mathrm{x})$, density function $\mathrm{r}_{\mathrm{i}}^{(l)}(\mathrm{x})$, Laplace Stieltje's transform $\mathrm{R}_{\mathrm{i}}^{(l) *}(\bullet), \mathrm{n}^{\text {th }}$ factorial moments $\beta_{\mathrm{i}, \mathrm{n}}^{(l)}$ and conditional completion rate $\beta_{\mathrm{i}}^{(l)}(\mathrm{x})=\mathrm{r}_{\mathrm{i}}^{(l)}(\mathrm{x}) /\left[1-\mathrm{r}_{\mathrm{i}}^{(l)}(\mathrm{x})\right]$, for $\mathrm{i}=1,2, \ldots, \mathrm{M}$ and $l=1,2$.

During type i service, if the server fails under mode 2 then the interrupted customer either remains in service position with 
probability $\tau_{\mathrm{i}}$ or leaves the system and keeps returning at times exponentially distributed with rate $\theta_{\mathrm{i}}$. As soon as the server is fixed, it continues the service of the interrupted customer or waits for the same customer to complete the remaining service. This waiting time of the server is referred as reserved time. The server is not allowed to accept new customer until the interrupted customer leaves the system.

\section{JOINT DISTRIBUTION OF THE SERVER STATE}

For $\mathrm{i}=1,2, \ldots, \mathrm{M}$, we define the server state $\mathrm{J}(\mathrm{t})$ as follows

$$
J(t)=\left\{\begin{array}{c}
0, \quad \text { server is idle } \\
i, \quad \text { server is busy in type i service } \\
M+i, \text { server failed under mode } 1 \text { during } i^{\text {th }} \text { type service is in setup time } \\
2 \mathrm{M}+\mathrm{i}, \text { server failed due to mode } 1 \text { during } \mathrm{i}^{\text {th }} \text { type service is under repair } \\
3 \mathrm{M}+\mathrm{i}, \text { server failed under mode } 2 \text { during } \mathrm{i}^{\text {th }} \text { type service is in setup time } \\
4 \mathrm{M}+\mathrm{i}, \text { server failed due to mode } 2 \text { during } \mathrm{i}^{\text {th }} \text { type service is under repair } \\
5 \mathrm{M}+\mathrm{i}, \text { server is under reserved time }
\end{array}\right.
$$

Let $X(t)$ denote the number of customers in the orbit. The state of the interrupted customer $\mathrm{J}^{*}(\mathrm{t})$ is defined as

$J^{*}(\mathrm{t})=$

0 , if the interrupted customer remains in service position

1 , if the interrupted customer leaves the service area

Define supplementary variable as

$\xi_{0}(\mathrm{t})=$ elapsed retrial time; $\xi_{1}(\mathrm{t})=$ elapsed service time $; \xi_{2}(\mathrm{t})$

$=$ elapsed setup time $; \xi_{3}(\mathrm{t})=$ elapsed repair time;

$\xi_{4}(\mathrm{t})=$ elapsed reserved time

The state of the system at time $t$ can be described by the Markov processes $\{\mathrm{N}(\mathrm{t}), \mathrm{t} \geq 0\}=\left\{\mathrm{J}(\mathrm{t}), \mathrm{J}^{*}(\mathrm{t}), \mathrm{X}(\mathrm{t}), \quad \xi_{0}(\mathrm{t}), \xi_{1}(\mathrm{t})\right.$, $\left.\xi_{2}(\mathrm{t}), \xi_{3}(\mathrm{t}), \xi_{4}(\mathrm{t}), \mathrm{t} \geq 0\right\}$.

Define the probability densities

$$
\begin{aligned}
& \mathrm{I}_{0}(\mathrm{t}) \quad=\mathrm{P}\{\mathrm{J}(\mathrm{t})=0, \mathrm{X}(\mathrm{t})=0\} \\
& \mathrm{I}_{\mathrm{n}}(\mathrm{x}, \mathrm{t}) \mathrm{dx} \quad=\mathrm{P}\left\{\mathrm{J}(\mathrm{t})=0, \mathrm{X}(\mathrm{t})=\mathrm{n}, \mathrm{x} \leq \xi_{0}(\mathrm{t})<\mathrm{x}+\mathrm{dx}\right\}, \mathrm{n} \geq 1 \\
& \text { For } \mathrm{n} \geq 0, \mathrm{i}=1,2, \ldots, \mathrm{M} \text { and } \mathrm{j}=0,1 \\
& \mathrm{P}_{\mathrm{i}, \mathrm{n}}(\mathrm{x}, \mathrm{t}) \mathrm{dx}=\mathrm{P}\left\{\mathrm{J}(\mathrm{t})=\mathrm{i}, \mathrm{X}(\mathrm{t})=\mathrm{n}, \mathrm{x} \leq \xi_{1}(\mathrm{t})<\mathrm{x}+\mathrm{dx}\right\} \\
& \mathrm{S}_{\mathrm{i}, \mathrm{n}}^{(1)}(\mathrm{x}, \mathrm{t}) \mathrm{dx}=\mathrm{P}\left\{\mathrm{J}(\mathrm{t})=\mathrm{M}+\mathrm{i}, \mathrm{X}(\mathrm{t})=\mathrm{n}, \mathrm{x} \leq \xi_{2}(\mathrm{t})<\mathrm{x}+\mathrm{dx}\right\} \\
& \mathrm{R}_{\mathrm{i}, \mathrm{n}}^{(1)}(\mathrm{x}, \mathrm{t}) \mathrm{dx} \quad=\mathrm{P}\left\{\mathrm{J}(\mathrm{t})=2 \mathrm{M}+\mathrm{i}, \mathrm{X}(\mathrm{t})=\mathrm{n}, \mathrm{x} \leq \xi_{3}(\mathrm{t})<\mathrm{x}+\mathrm{dx}\right\} \\
& S_{i, j, n}^{(2)}(x, y, t) d x d y=P\left\{J(t)=3 M+i, J^{*}(t)=j, X(t)=n,\right. \\
& \left.\mathrm{x} \leq \xi_{1}(\mathrm{t})<\mathrm{x}+\mathrm{dx}, \mathrm{y} \leq \xi_{2}(\mathrm{t})<\mathrm{y}+\mathrm{dy}\right\} \\
& R_{i, j, n}^{(2)}(x, y, t) d x d y=P\left\{J(t)=4 M+i, J^{*}(t)=j, X(t)=n,\right. \\
& \left.\mathrm{x} \leq \xi_{1}(\mathrm{t})<\mathrm{x}+\mathrm{dx}, \mathrm{y} \leq \xi_{3}(\mathrm{t})<\mathrm{y}+\mathrm{dy}\right\} \\
& \mathrm{Q}_{\mathrm{i}, \mathrm{n}}(\mathrm{x}, \mathrm{y}, \mathrm{t}) \mathrm{dxdy} \quad=\mathrm{P}\{\mathrm{J}(\mathrm{t})=5 \mathrm{M}+\mathrm{i}, \mathrm{X}(\mathrm{t})=\mathrm{n} \text {, } \\
& \left.\mathrm{x} \leq \xi_{1}(\mathrm{t})<\mathrm{x}+\mathrm{dx}, \mathrm{y} \leq \xi_{4}(\mathrm{t})<\mathrm{y}+\mathrm{dy}\right\}
\end{aligned}
$$

The governing equations of the model under steady state are

$$
\lambda^{+} I_{0}=\sum_{i=1}^{M}\left[\int_{0}^{\infty} P_{i, 0}(x) \mu_{i}(x) d x+\int_{0}^{\infty} R_{i, 0}^{(1)}(x) \beta_{i}^{(1)}(x) d x\right]
$$

$$
\begin{aligned}
& \frac{d}{d x} I_{n}(x)=-\left(\lambda^{+}+\eta(x)\right) I_{n}(x), n \geq 1 \\
& \frac{d}{d x} P_{i, n}(x)=-\left(\lambda^{+}+\lambda^{-}+\alpha_{i}+\mu_{i}(x)\right) P_{i, n}(x)+\lambda^{+} \sum_{k=1}^{n} C_{k} P_{i, n-k}(x)+ \\
& \quad \int_{i, 0, n}^{\infty} R_{i}^{(2)}(x, y) \beta_{i}^{(2)}(y) d y+\theta_{i} \int_{0}^{\infty} Q_{i, n}(x, y) d y, \quad n \geq 0, i=1,2, \ldots, M \\
& \frac{d}{d x} S_{i, n}^{(1)}(x)=-\left(\lambda^{+}+\phi_{i}^{(1)}(x)\right) S_{i, n}^{(1)}(x)+\lambda^{+} \sum_{k=1}^{n} C_{k} S_{i, n-k}^{(1)}(x), \\
& \frac{d}{d x} R_{i, n}^{(1)}(x)=-\left(\lambda^{+}+\beta_{i}^{(1)}(x)\right) R_{i, n}^{(1)}(x)+\lambda^{+} \sum_{k=1}^{n} C_{k} R_{i, n-k}^{(1)}(x), \\
& \frac{d}{d y} S_{i, j, n}^{(2)}(x, y)=-\left(\lambda^{+}+\phi_{i}^{(2)}(y)\right) S_{i, j, n}^{(2)}(x, y)+\lambda^{+} \sum_{k=1}^{n} C_{k} S_{i, j, n-k}^{(2)}(x, y), \\
& n \geq 0, i=1,2, \ldots, M, j=0,1 \\
& \frac{d}{d y} Q_{i, n}(x, y)=-\left(\lambda^{+}+\theta_{i}\right) Q_{i, n}(x, y)+\lambda^{+} \sum_{k=1}^{n} C_{k} C_{i, j, n} Q_{i, n-k}(x, y)=-\left(\lambda^{+}+\beta_{i}^{(2)}(y)\right) R_{i, j, n}^{(2)}(x, y)+\lambda^{+} \sum_{i=1}^{n} C_{k} R_{i, j, n-k}^{(2)}(x, y), \\
& n \geq 0, i=1,2, \ldots, M
\end{aligned}
$$

with boundary conditions

$$
\begin{aligned}
& \mathrm{I}_{\mathrm{n}}(0)=\sum_{\mathrm{i}=1}^{\mathrm{M}}\left[\int_{0}^{\infty} \mathrm{P}_{\mathrm{i}, \mathrm{n}}(\mathrm{x}) \mu_{\mathrm{i}}(\mathrm{x}) \mathrm{dx}+\int_{0}^{\infty} \mathrm{R}_{\mathrm{i}, \mathrm{n}}^{(1)}(\mathrm{x}) \beta_{\mathrm{i}}^{(1)}(\mathrm{x}) \mathrm{dx}\right], \mathrm{n} \geq 1 \\
& \mathrm{P}_{\mathrm{i}, 0}(0)=\mathrm{p}_{\mathrm{i}}\left[\lambda^{+} \mathrm{c}_{1} \mathrm{I}_{0}+\int_{0}^{\infty} \mathrm{I}_{1}(\mathrm{x}) \eta(\mathrm{x}) \mathrm{dx}\right], \mathrm{i}=1,2, \ldots, \mathrm{M} \\
& \mathrm{P}_{\mathrm{i}, \mathrm{n}}(0)=\mathrm{p}_{\mathrm{i}}\left[\lambda^{+} \mathrm{c}_{\mathrm{n}+1} \mathrm{I}_{0}+\int_{0}^{\infty} \mathrm{I}_{\mathrm{n}+1}(\mathrm{x}) \eta(\mathrm{x}) \mathrm{dx}+\lambda^{+} \sum_{\mathrm{k}=1}^{\mathrm{n}} \mathrm{C}_{\mathrm{k}} \int_{0}^{\infty} \mathrm{I} \mathrm{n}-\mathrm{k}+1\right. \\
& \mathrm{n} \geq 1, \mathrm{i}=1,2, \ldots, \mathrm{M}
\end{aligned}
$$$$
S_{i, n}^{(1)}(0)=\lambda^{-} \int_{i, n}^{\infty} P_{i}(x) d x, n \geq 0, i=1,2, \ldots, M
$$$$
0
$$$$
\mathrm{R}_{\mathrm{i}, \mathrm{n}}^{(1)}(0)=\int_{0}^{\infty} \mathrm{S}_{\mathrm{i}, \mathrm{n}}^{(1)}(\mathrm{x}) \phi_{\mathrm{i}}^{(1)}(\mathrm{x}) \mathrm{dx}, \mathrm{n} \geq 0, \mathrm{i}=1,2, \ldots, \mathrm{M}
$$$$
\mathrm{S}_{\mathrm{i}, 0, \mathrm{n}}^{(2)}(\mathrm{x}, 0)=\tau_{\mathrm{i}} \alpha_{\mathrm{i}} \mathrm{P}_{\mathrm{i}, \mathrm{n}}(\mathrm{x}), \mathrm{n} \geq 0, \mathrm{i}=1,2, \ldots, \mathrm{M}
$$

$\mathrm{S}_{\mathrm{i}, 1, \mathrm{n}}^{(2)}(\mathrm{x}, 0)=\left(1-\tau_{\mathrm{i}}\right) \alpha_{\mathrm{i}} \mathrm{P}_{\mathrm{i}, \mathrm{n}}(\mathrm{x}), \mathrm{n} \geq 0, \mathrm{i}=1,2, \ldots, \mathrm{M}$

$\mathrm{R}_{\mathrm{i}, \mathrm{j}, \mathrm{n}}^{(2)}(\mathrm{x}, 0)=\int_{0}^{\infty} \mathrm{S}_{\mathrm{i}, \mathrm{j}, \mathrm{n}}^{(2)}(\mathrm{x}, \mathrm{y}) \phi_{\mathrm{i}}^{(2)}(\mathrm{y}) \mathrm{dy}, \mathrm{n} \geq 0, \mathrm{i}=1,2, \ldots, \mathrm{M}, \mathrm{j}=0,1$

$$
\mathrm{Q}_{\mathrm{i}, \mathrm{n}}(\mathrm{x}, 0)=\int_{0}^{\infty} \mathrm{R}_{\mathrm{i}, 1, \mathrm{n}}^{(2)}(\mathrm{x}, \mathrm{y}) \beta_{\mathrm{i}}^{(2)}(\mathrm{y}) \mathrm{dy}, \mathrm{n} \geq 0, \mathrm{i}=1,2, \ldots, \mathrm{M}
$$

.


The normalizing condition is

$I_{0}+\mathrm{I}(1)+\sum_{i=1}^{M}\left[P_{i}(1)+S_{i}^{(1)}(1)+R_{i}^{(1)}(1)+S_{i, 0}^{(2)}(1)+S_{i, 1}^{(2)}(1)+R_{i, 0}^{(2)}(1)+R_{i, 1}^{(2)}(1)+Q_{i}(1)\right]=1$

Define the joint probability generating functions

$\mathrm{I}(\mathrm{x}, \mathrm{z})=\sum_{\mathrm{n}=1}^{\infty} \mathrm{I}_{\mathrm{n}}(\mathrm{x}) \mathrm{z}^{\mathrm{n}}$,

$P_{i}(x, z)=\sum_{n=0}^{\infty} P_{i, n}(x) z^{n}$,

$S_{i}^{(1)}(x, z)=\sum_{n=0}^{\infty} S_{i, n}^{(1)}(x) z^{n}$,

$\mathrm{R}_{\mathrm{i}}^{(1)}(\mathrm{x}, \mathrm{z})=\sum_{\mathrm{n}=0}^{\infty} \mathrm{R}_{\mathrm{i}, \mathrm{n}}^{(1)}(\mathrm{x}) \mathrm{z}^{\mathrm{n}}$,

$S_{i, j}^{(2)}(x, y, z)=\sum_{n=0}^{\infty} S_{i, j, n}^{(2)}(x, y) z^{n}$,

$R_{i, j}^{(2)}(x, y, z)=\sum_{n=0}^{\infty} R_{i, j, n}^{(2)}(x, y) z^{n}$,

$\mathrm{Q}_{\mathrm{i}}(\mathrm{x}, \mathrm{y}, \mathrm{z})=\sum_{\mathrm{n}=0}^{\infty} \mathrm{Q}_{\mathrm{i}, \mathrm{n}}(\mathrm{x}, \mathrm{y}) \mathrm{z}^{\mathrm{n}}, \mathrm{i}=1$ to $\mathrm{M}, \mathrm{j}=0,1$.

Multiplying equations (2) to (17) by $\mathrm{z}^{\mathrm{n}}$ and summing over for all possible values of $n$, we get

$\left(\frac{\mathrm{d}}{\mathrm{dx}}+\lambda^{+}+\eta(\mathrm{x})\right) \mathrm{I}(\mathrm{x}, \mathrm{z})=0$

$\left(\frac{\mathrm{d}}{\mathrm{dx}}+\lambda^{+}+\lambda^{-}+\alpha_{i}+\mu_{\mathrm{i}}(\mathrm{x})-\lambda^{+} \mathrm{C}(\mathrm{z})\right) \mathrm{P}^{(\mathrm{i})}(\mathrm{x}, \mathrm{z})=$

$\int_{0}^{\infty} \mathrm{R}_{\mathrm{i}, 0}^{(2)}(\mathrm{x}, \mathrm{y}, \mathrm{z}) \mathrm{\beta}_{\mathrm{i}}^{(2)}(\mathrm{y}) \mathrm{dy}+\theta_{\mathrm{i}} \int_{0}^{\infty} \mathrm{Q}_{\mathrm{i}}(\mathrm{x}, \mathrm{y}, \mathrm{z}) \mathrm{dy}, \mathrm{i}=1,2, \ldots, \mathrm{M}$

$\left(\frac{\mathrm{d}}{\mathrm{dx}}+\lambda^{+}+\phi_{\mathrm{i}}^{(1)}(\mathrm{x})-\lambda^{+} \mathrm{C}(\mathrm{z})\right) \mathrm{S}_{\mathrm{i}}^{(1)}(\mathrm{x}, \mathrm{z})=0, \mathrm{i}=1,2, \ldots, \mathrm{M}$

$\left(\frac{\mathrm{d}}{\mathrm{dx}}+\lambda^{+}+\beta_{\mathrm{i}}^{(1)}(\mathrm{x})-\lambda^{+} \mathrm{C}(\mathrm{z})\right) \mathrm{R}_{\mathrm{i}}^{(1)}(\mathrm{x}, \mathrm{z})=0, \mathrm{i}=1,2, \ldots, \mathrm{M}$

$\left(\frac{\mathrm{d}}{\mathrm{dy}}+\lambda^{+}+\phi_{\mathrm{i}}^{(2)}(\mathrm{y})-\lambda^{+} \mathrm{C}(\mathrm{z})\right) \mathrm{S}_{\mathrm{i}, \mathrm{j}}^{(2)}(\mathrm{x}, \mathrm{y}, \mathrm{z})=0$,

$$
\mathrm{i}=1,2, \ldots, \mathrm{M}, \mathrm{j}=0,1
$$

$\left(\frac{\mathrm{d}}{\mathrm{dy}}+\lambda^{+}+\beta_{\mathrm{i}}^{(2)}(\mathrm{y})-\lambda^{+} \mathrm{C}(\mathrm{z})\right) \mathrm{R}_{\mathrm{i}, \mathrm{j}}^{(2)}(\mathrm{x}, \mathrm{y}, \mathrm{z})=0$,

$$
\mathrm{i}=1,2, \ldots, \mathrm{M}, \mathrm{j}=0,1
$$

$\left(\frac{d}{d y}+\lambda^{+}+\theta_{i}-\lambda^{+} C(z)\right) Q_{i}(x, y, z)=0, i=1,2, \ldots, M$

$\mathrm{I}(0, \mathrm{z})=\sum_{\mathrm{i}=1}^{\mathrm{M}}\left[\int_{0}^{\infty} \mathrm{P}_{\mathrm{i}}(\mathrm{x}, \mathrm{z}) \mu_{\mathrm{i}}(\mathrm{x}) \mathrm{dx}+\int_{0}^{\infty} \mathrm{R}_{\mathrm{i}}^{(1)}(\mathrm{x}, \mathrm{z}) \beta_{\mathrm{i}}^{(1)}(\mathrm{x}) \mathrm{dx}\right]-\lambda^{+} \mathrm{I}_{0}$

$\left.\mathrm{P}_{\mathrm{i}}(0, \mathrm{z})=\frac{\mathrm{p}_{\mathrm{i}}}{\mathrm{z}}\left[\lambda^{+} \mathrm{C}(\mathrm{z}) \mathrm{I}_{0}+\int_{0}^{\infty} \mathrm{I}(\mathrm{x}, \mathrm{z}) \eta\right) \eta(\mathrm{x})+\lambda^{+} \mathrm{C}(\mathrm{z}) \int_{0}^{\infty} \mathrm{I}(\mathrm{x}, \mathrm{z}) \mathrm{dx}\right], \mathrm{i}=1,2, \ldots, \mathrm{M}$

$S_{i}^{(1)}(0, z)=\lambda^{-} \int_{0}^{\infty} P_{i}(x, z) d x, i=1,2, \ldots, M$
$\mathrm{R}_{\mathrm{i}}^{(1)}(0, \mathrm{z})=\int^{\infty} \mathrm{S}_{\mathrm{i}}^{(1)}(\mathrm{x}, \mathrm{z}) \phi_{\mathrm{i}}^{(1)}(\mathrm{x}) \mathrm{dx}, \mathrm{i}=1,2, \ldots, \mathrm{M}$

$\mathrm{S}_{\mathrm{i}, 0}^{(2)}(\mathrm{x}, 0, \mathrm{z})=\tau_{\mathrm{i}} \alpha_{\mathrm{i}} \mathrm{P}_{\mathrm{i}}(\mathrm{x}, \mathrm{z}), \mathrm{i}=1,2, \ldots, \mathrm{M}$

$S_{i, 1}^{(2)}(x, 0, z)=\left(1-\tau_{i}\right) \alpha_{i} P_{i}(x, z), i=1,2, \ldots, M$

$\mathrm{R}_{\mathrm{i}, \mathrm{j}}^{(2)}(\mathrm{x}, 0, \mathrm{z})=\int_{0}^{\infty} \mathrm{S}_{\mathrm{i}, \mathrm{j}}^{(2)}(\mathrm{x}, \mathrm{y}, \mathrm{z}) \phi_{\mathrm{i}}^{(2)}(\mathrm{y}) \mathrm{dy}, \mathrm{i}=1,2, \ldots, \mathrm{M}, \mathrm{j}=0,1$

$\mathrm{Q}_{\mathrm{i}}(\mathrm{x}, 0, \mathrm{z})=\int_{0}^{\infty} \mathrm{R}_{\mathrm{i}, 1}^{(2)}(\mathrm{x}, \mathrm{y}, \mathrm{z}) \beta_{\mathrm{i}}^{(2)}(\mathrm{y}) \mathrm{dy}, \mathrm{i}=1,2, \ldots, \mathrm{M}$

Solving the partial differential equations (19),(21) - (25), we get

$$
\begin{aligned}
& I(x, z)=I(0, z) e^{-\lambda^{+} x}[1-A(x)] \\
& S_{i}^{(1)}(x, z)=S_{i}^{(1)}(0, z) e^{-\lambda^{+}(1-C(z)) x}\left[1-S_{i}^{(1)}(x)\right] \\
& R_{i}^{(1)}(x, z)=R_{i}^{(1)}(0, z) e^{\left.-\lambda^{+}(1-C(z)) x_{\left[1-R_{i}\right.}^{(1)}(x)\right]} \\
& S_{i, j}^{(2)}(x, y, z)=S_{i, j}^{(2)}(x, 0, z) e^{\left.-\lambda^{+}(1-C(z)) y_{\left[1-S_{i}\right.}^{(2)}(y)\right]}, \\
& R_{i, j}^{(2)}(x, y, z)=R_{i, j}^{(2)}(x, 0, z) e^{-\lambda^{+}(1-C(z)) y}{ }_{\left[1-R_{i}^{(2)}(y)\right]} \\
& Q_{i}(x, y, z)=Q_{i}(x, 0, z) e^{-\left[\lambda^{+}(1-C(z))+\theta_{i}\right] y}, i=1,2, \ldots, M, M
\end{aligned}
$$

Using equations (30), (31) and (37) in equation (32) we obtain,

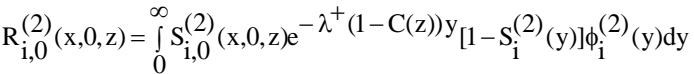

$$
\begin{aligned}
& =\mathrm{S}_{\mathrm{i}, 0}^{(2)}(\mathrm{x}, 0, \mathrm{z}) \mathrm{S}_{\mathrm{i}}^{(2)}{ }^{*}[\mathrm{~h}(\mathrm{z})]
\end{aligned}
$$

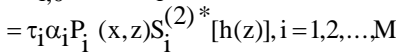

$\mathrm{R}_{\mathrm{i}, 1}^{(2)}(\mathrm{x}, 0, \mathrm{z})=\left(1-\tau_{\mathrm{i}}\right) \alpha_{\mathrm{i}} \mathrm{P}_{\mathrm{i}}(\mathrm{x}, \mathrm{z}) \mathrm{S}_{\mathrm{i}}^{(2) *}[\mathrm{~h}(\mathrm{z})], \mathrm{i}=1,2, \ldots, \mathrm{M}$ where, $\mathrm{h}(\mathrm{z})=\lambda^{+}-\lambda^{+} \mathrm{C}(\mathrm{z})$

Using equations (38) and (41), equation (33) becomes $\mathrm{Q}_{\mathbf{i}}(\mathrm{x}, 0, \mathrm{z})=\left(1-\tau_{\mathrm{i}}\right) \alpha_{\mathrm{i}} \mathrm{P}_{\mathrm{i}}(\mathrm{x}, \mathrm{z}) \mathrm{S}_{\mathrm{i}}^{(2)}{ }^{*}[\mathrm{~h}(\mathrm{z})] \mathrm{R}_{\mathrm{i}}^{(2)}{ }^{*}[\mathrm{~h}(\mathrm{z})], \mathrm{i}=1,2, \ldots, \mathrm{M}$.

Substituting the expressions of $\mathrm{R}_{\mathrm{i}, 0}^{(2)}(\mathrm{x}, \mathrm{y}, \mathrm{z})$ and $\mathrm{Q}_{\mathrm{i}}(\mathrm{x}, \mathrm{y}, \mathrm{z})$ in equation (20) and solving, we get

$P_{i}(x, z)=P_{i}(0, z) e^{-(g(z)) x}\left[1-B_{i}(x)\right], i=1,2, \ldots, M$

where

$g(z)=h(z)+\lambda^{-}+\alpha_{i}-\alpha_{i} S_{i}^{(2) *}(h(z)) R_{i}^{(2)} *(h(z))\left(\frac{h(z) \tau_{i}+\theta_{i}}{h(z)+\theta_{i}}\right)$ 
Using equations (28),(29),(35),(36) and (43) in equation (26), we get

$\mathrm{I}(0, \mathrm{z})=\sum_{\mathrm{i}=1}^{\mathrm{M}} \mathrm{P}_{\mathrm{i}}(0, \mathrm{z})\left[\mathrm{B}_{\mathrm{i}}^{*}(\mathrm{~g}(\mathrm{z}))+\lambda^{-} \overline{\mathrm{B}}_{\mathrm{i}}^{*}(\mathrm{~g}(\mathrm{z})) \mathrm{S}_{\mathrm{i}}^{(1) *}(\mathrm{~h}(\mathrm{z})) \mathrm{R}_{\mathrm{i}}^{(1)}{ }^{*}(\mathrm{~h}(\mathrm{z}))\right]-\lambda^{+} \mathrm{I}_{0}$

Using equations (34) and (44) in equation (27) and on simplifying we get

$\mathrm{P}_{\mathrm{i}}(0, \mathrm{z})=\mathrm{I}_{0} \lambda^{+} \mathrm{p}_{\mathrm{i}} \mathrm{A}^{*}\left(\lambda^{+}\right) \mathrm{g}(\mathrm{z})(\mathrm{C}(\mathrm{z})-1) / \mathrm{D}(\mathrm{z}), \mathrm{i}=1,2, \ldots \mathrm{M}$

Substituting the expression of $\mathrm{P}_{\mathrm{i}}(0, \mathrm{z})$ in equation (44), we obtain

$$
\mathrm{I}(0, \mathrm{z})=\lambda^{+} \mathrm{I}_{0}\left[\begin{array}{l}
\mathrm{C}(\mathrm{z}) \sum_{\mathrm{i}=1}^{\mathrm{M}} \mathrm{p}_{\mathrm{i}}\left[\mathrm{g}(\mathrm{z}) \mathrm{B}_{\mathrm{i}}^{*}(\mathrm{~g}(\mathrm{z}))+\right. \\
\lambda^{-}\left(1-\mathrm{B}_{\mathrm{i}}^{*}(\mathrm{~g}(\mathrm{z})) \mathrm{S}_{\mathrm{i}}^{(1)}{ }^{*}(\mathrm{~h}(\mathrm{z})) \mathrm{R}_{\mathrm{i}}^{(1)}{ }^{*}(\mathrm{~h}(\mathrm{z}))\right]-\mathrm{zg}(\mathrm{z})
\end{array}\right] / \mathrm{D}(\mathrm{z})
$$

where $\mathrm{D}(\mathrm{z})=$

$$
\begin{array}{r}
\left.\mathrm{zg}(\mathrm{z})-\left[\mathrm{A}^{*}\left(\lambda^{+}\right)+\mathrm{C}(\mathrm{z})\right)\left(1-\mathrm{A}^{*}\left(\lambda^{+}\right)\right)\right] \sum_{\mathrm{i}=1}^{\mathrm{M}} \mathrm{p}_{\mathrm{i}}\left[\mathrm{g}(\mathrm{z}) \mathrm{B}_{\mathrm{i}}^{*}(\mathrm{~g}(\mathrm{z}))+\right. \\
\lambda^{-}\left(1-\mathrm{B}_{\mathrm{i}}^{*}(\mathrm{~g}(\mathrm{z})) \mathrm{S}_{\mathrm{i}}^{(1) *}(\mathrm{~h}(\mathrm{z})) \mathrm{R}_{\mathrm{i}}^{(1) *}(\mathrm{~h}(\mathrm{z}))\right]
\end{array}
$$

Using equation (46) in equation (34) and integrating with respect to $\mathrm{x}$ from 0 to $\infty$, we get

$$
\mathrm{I}(\mathrm{z})=\mathrm{I}_{0}\left(1-\mathrm{A}^{*}\left(\lambda^{+}\right)\right)\left[\begin{array}{l}
\mathrm{C}(\mathrm{z}) \sum_{\mathrm{i}=1}^{\mathrm{M}} \mathrm{p}_{\mathrm{i}}\left[\mathrm{g}(\mathrm{z}) \mathrm{B}_{\mathrm{i}}^{*}(\mathrm{~g}(\mathrm{z}))+\right. \\
\lambda^{-}\left(1-\mathrm{B}_{\mathrm{i}}^{*}(\mathrm{~g}(\mathrm{z})) \mathrm{S}_{\mathrm{i}}^{(1) *}(\mathrm{~h}(\mathrm{z})) \mathrm{R}_{\mathrm{i}}^{(1) *}(\mathrm{~h}(\mathrm{z}))\right]-\mathrm{zg}(\mathrm{z})
\end{array}\right] / \mathrm{D}(\mathrm{z})
$$

Using equation (45) in equation (43) and integrating with respect to $\mathrm{x}$ from 0 to $\infty$, we get

$$
\mathrm{P}_{\mathrm{i}}(\mathrm{z})=\mathrm{I}_{0} \lambda^{+} \mathrm{p}_{\mathrm{i}} \mathrm{A}^{*}\left(\lambda^{+}\right)(\mathrm{C}(\mathrm{z})-1)\left[1-\mathrm{B}_{\mathrm{i}}^{*}(\mathrm{~g}(\mathrm{z}))\right] / \mathrm{D}(\mathrm{z}), \mathrm{i}=1,2, \ldots, \mathrm{M}
$$

Using equations (28),(35) and (47) and integrating with respect to $\mathrm{x}$ from 0 to $\infty$, yields

$\mathrm{S}_{\mathrm{i}}^{(1)}(\mathrm{z})=-\mathrm{I}_{0} \lambda^{-} \mathrm{p}_{\mathrm{i}} \mathrm{A}^{*}\left(\lambda^{+}\right)\left[1-\mathrm{B}_{\mathrm{i}}^{*}(\mathrm{~g}(\mathrm{z}))\right]\left[1-\mathrm{S}_{\mathrm{i}}^{(1) *}(\mathrm{~h}(\mathrm{z}))\right] / \mathrm{D}(\mathrm{z}), \mathrm{i}=1,2, \ldots, \mathrm{M}$

Using equations(29) and (49) in equation (36) and integrating with respect to $\mathrm{x}$ from 0 to $\infty$, yields

$$
\begin{aligned}
& \mathrm{R}_{\mathrm{i}}^{(1)}(\mathrm{z})= \\
& -\mathrm{I}_{0} \lambda^{-} \mathrm{p}_{\mathrm{i}} \mathrm{A}^{*}\left(\lambda^{+}\right)\left[1-\mathrm{B}_{\mathrm{i}}^{*}(\mathrm{~g}(\mathrm{z}))\right]\left[1-\mathrm{R}_{\mathrm{i}}^{(1) *}(\mathrm{~h}(\mathrm{z}))\right] \mathrm{S}_{\mathrm{i}}^{(1)}{ }^{*}(\mathrm{~h}(\mathrm{z})) / \mathrm{D}(\mathrm{z}), \\
& \mathrm{i}=1,2, \ldots, \mathrm{M}
\end{aligned}
$$

Using equations (30),(31) and (47) and substituting the result in equation (37)-(39), integrating with respect to $\mathrm{x}$ and $\mathrm{y}$ from 0 to $\infty$ we obtain

$$
\begin{aligned}
& \mathrm{S}_{\mathrm{i}, 0}^{(2)}(\mathrm{z})=-\mathrm{I}_{0} \tau_{\mathrm{i}} \mathrm{p}_{\mathrm{i}} \alpha_{\mathrm{i}} \mathrm{A}^{*}\left(\lambda^{+}\right)\left[1-\mathrm{B}_{\mathrm{i}}^{*}(\mathrm{~g}(\mathrm{z}))\right]\left[1-\mathrm{S}_{\mathrm{i}}^{(2)}{ }^{*}(\mathrm{~h}(\mathrm{z}))\right] / \mathrm{D}(\mathrm{z}), \\
& \mathrm{i}=1,2, \ldots, \mathrm{M} \\
& \mathrm{S}_{\mathrm{i}, 1}^{(2)}(\mathrm{z})=-\mathrm{I}_{0}\left(1-\tau_{\mathrm{i}}\right) \mathrm{p}_{\mathrm{i}} \alpha_{\mathrm{i}} \mathrm{A}^{*}\left(\lambda^{+}\right)\left[1-\mathrm{B}_{\mathrm{i}}^{*}(\mathrm{~g}(\mathrm{z}))\right]\left[1-\mathrm{S}_{\mathrm{i}}^{(2)}(\mathrm{h}(\mathrm{z}))\right] / \mathrm{D}(\mathrm{z}), \\
& \mathrm{i}=1,2, \ldots, \mathrm{M} \\
& \mathrm{R}_{\mathrm{i}, 0}^{(2)}(\mathrm{z})=-\mathrm{I}_{0} \tau_{\mathrm{i}} \mathrm{p}_{\mathrm{i}} \alpha_{\mathrm{i}} \mathrm{A}^{*}\left(\lambda^{+}\right)\left[1-\mathrm{B}_{\mathrm{i}}^{*}(\mathrm{~g}(\mathrm{z})] \mathrm{S}_{\mathrm{i}}^{(2)}{ }^{*}(\mathrm{~h}(\mathrm{z}))\left[1-\mathrm{R}_{\mathrm{i}}^{(2){ }^{*}}(\mathrm{~h}(\mathrm{z})] / \mathrm{D}(\mathrm{z}),\right.\right. \\
& \mathrm{i}=1,2, \ldots, \mathrm{M}
\end{aligned}
$$

$$
\begin{gathered}
\mathrm{R}_{\mathrm{i}, 1}^{(2)}(\mathrm{z})=-\mathrm{I}_{0}\left(1-\tau_{\mathrm{i}}\right) \mathrm{p}_{\mathrm{i}} \alpha_{\mathrm{i}} \mathrm{A}^{*}\left(\lambda^{+}\right)\left[1-\mathrm{B}_{\mathrm{i}}^{*}(\mathrm{~g}(\mathrm{z})] \mathrm{S} \mathrm{S}_{\mathrm{i}}^{(2) *}(\mathrm{~h}(\mathrm{z}))\left[1-\mathrm{R}_{\mathrm{i}}^{(2) *}(\mathrm{~h}(\mathrm{z}))\right] / \mathrm{D}(\mathrm{z}), \quad(54)\right. \\
\mathrm{i}=1,2, \ldots, \mathrm{M} \\
\mathrm{Q}_{\mathrm{i}}(\mathrm{z})=-\mathrm{I}_{0}\left(1-\tau_{\mathrm{i}}\right) \mathrm{p}_{\mathrm{i}} \alpha_{\mathrm{i}} \mathrm{A}^{*}\left(\lambda^{+}\right) \mathrm{h}(\mathrm{z})\left[1-\mathrm{B}_{\mathrm{i}}^{*}(\mathrm{~g}(\mathrm{z})) \mathrm{S}_{\mathrm{i}}^{(2)}{ }^{*}(\mathrm{~h}(\mathrm{z})) \mathrm{R}_{\mathrm{i}}^{(2) *}(\mathrm{~h}(\mathrm{z})) /\left[\mathrm{h}(\mathrm{z})+\theta_{\mathrm{i}}\right] \mathrm{D}(\mathrm{z}),\right. \\
\mathrm{i}=1,2, \ldots, \mathrm{M}
\end{gathered}
$$

Using normalizing condition $\mathrm{I}_{0}$ is obtained as

$$
\mathrm{I}_{0}=\frac{\lambda^{-}\left(1-\mathrm{m}_{1}\left(1-\mathrm{A}^{*}\left(\lambda^{+}\right)\right)\right)-\lambda^{+} \mathrm{m}_{1} \sum_{\mathrm{i}=1}^{\mathrm{M}} \mathrm{p}_{\mathrm{i}}\left(1-\mathrm{B}_{\mathrm{i}}^{*}\left(\lambda^{-}\right)\right)\left(\lambda^{-}\left(\phi_{\mathrm{i}, 1}^{(1)}+\beta_{\mathrm{i}, 1}^{(1)}\right)+\mathrm{T}_{1}\right)}{\lambda^{-} \mathrm{A}^{*}\left(\lambda^{+}\right)}
$$

The probability generating function $P_{q}(z)$ of the number of customers in the orbit is

$\mathrm{P}_{\mathrm{q}}(\mathrm{z})=\mathrm{I}_{0} \mathrm{~A}^{*}\left(\lambda^{+}\right) \mathrm{g}(\mathrm{z})(\mathrm{z}-1) / \mathrm{D}(\mathrm{z})$

The probability generating function $P_{s}(z)$ of the number of customers in the system is

$$
\mathrm{P}_{\mathrm{S}}(\mathrm{z})=\mathrm{I}_{0} \mathrm{~A}^{*}\left(\lambda^{+}\right)(\mathrm{z}-1)\left(\mathrm{g}(\mathrm{z}) \sum_{\mathrm{i}=1}^{\mathrm{M}} \mathrm{p}_{\mathrm{i}} \mathrm{B}_{\mathrm{i}}^{*}(\mathrm{~g}(\mathrm{z}))+\lambda^{-} \sum_{\mathrm{i}=1}^{\mathrm{M}} \mathrm{p}_{\mathrm{i}}\left(1-\mathrm{B}_{\mathrm{i}}^{*}(\mathrm{~g}(\mathrm{z}))\right) / \mathrm{D}(\mathrm{z})\right.
$$

\section{PERFORMANCE MEASURES}

The steady state probability that the system is empty is $\mathrm{I}_{0}$. The steady state probability that the server is idle in nonempty system is

$$
=\frac{\left(1-\mathrm{A}^{*}\left(\lambda^{+}\right)\right)\left[\lambda^{-}\left(\mathrm{m}_{1}-1\right)+\lambda^{+} \mathrm{m}_{1} \sum_{\mathrm{i}=1}^{\mathrm{M}} \mathrm{p}_{\mathrm{i}}\left(1-\mathrm{B}_{\mathrm{i}}^{*}\left(\lambda^{-}\right)\right)\left(\lambda^{-}\left(\phi_{i, 1}^{(1)}+\beta_{i, 1}^{(1)}\right)+\mathrm{T}_{1}\right)\right]}{\lambda^{-} \mathrm{A}^{*}\left(\lambda^{+}\right)}
$$

The steady state probability that the server is busy is

$\mathrm{P}=\sum_{\mathrm{i}=1}^{\mathrm{M}} \mathrm{P}_{\mathrm{i}}=\lambda^{+} \mathrm{m}_{1} \sum_{\mathrm{i}=1}^{\mathrm{M}} \mathrm{p}_{\mathrm{i}}\left(1-\mathrm{B}_{\mathrm{i}}^{*}\left(\lambda^{-}\right)\right) / \lambda^{-}$

The steady state probability that the server is under mode 1 repair

$$
\mathrm{H}=\underset{\mathrm{i}=1}{\mathrm{M}=1}\left(\mathrm{~S}_{\mathrm{i}}^{(1)}+\mathrm{S}_{\mathrm{i}}^{(1)}+\mathrm{R}_{\mathrm{i}}^{(1)}+\mathrm{R}_{\mathrm{i}}^{(1)}\right)=\lambda^{+} \mathrm{m}_{1} \sum_{\mathrm{i}=1}^{\mathrm{M}} \mathrm{p}_{\mathrm{i}}\left(1-\mathrm{B}_{\mathrm{i}}^{*}\left(\lambda^{-}\right)\right)\left(\varphi_{i, 1}^{(1)}+\beta_{\mathrm{i}, 1}^{(1)}\right)
$$

The steady state probability that the server is under mode 2 repair

$$
\begin{aligned}
F & =\sum_{i=1}^{M}\left(S_{i, 0}^{(2)}+S_{i, 1}^{(2)}+R_{i, 0}^{(2)}+R_{i, 1}^{(2)}\right) \\
& =\lambda^{+} m_{1} \sum_{i=1}^{M} \alpha_{i} p_{i}\left(1-B_{i}^{*}\left(\lambda^{-}\right)\right)\left(\phi_{i, 1}^{(2)}+\beta_{i, 1}^{(2)}\right) / \lambda^{-}
\end{aligned}
$$

The steady state probability that the server is under reserved time is

$$
\mathrm{Q}=\sum_{\mathrm{i}=1}^{\mathrm{M}} \mathrm{Q}_{\mathrm{i}}=\lambda^{+} \mathrm{m}_{1} \sum_{\mathrm{i}=1}^{\mathrm{M}} \mathrm{p}_{\mathrm{i}}\left(1-\mathrm{B}_{\mathrm{i}}^{*}\left(\lambda^{-}\right)\right) \alpha_{\mathrm{i}}\left(1-\tau_{\mathrm{i}}\right) / \lambda^{-} \theta_{\mathrm{i}}
$$

The mean number of customers in the orbit $\mathrm{L}_{\mathrm{q}}$ is given by $\mathrm{L}_{\mathrm{q}}=\mathrm{P}_{\mathrm{q}}^{\prime}(1)=\frac{\left[\mathrm{D}^{\prime}(1) \mathrm{Nr}^{\prime \prime}(1)-\mathrm{Nr}^{\prime}(1) \mathrm{D}^{\prime \prime}(1)\right]}{2 \mathrm{D}^{\prime}(1)^{2}}$

Let $\operatorname{Nr}(\mathrm{z})$ and $\mathrm{D}(\mathrm{z})$ represent the numerator and denominator of $\mathrm{P}_{\mathrm{q}}(\mathrm{z})$.

where,

$\mathrm{Nr}^{\prime}(1)=\mathrm{I}_{0} \lambda^{-} \mathrm{A}^{*}\left(\lambda^{+}\right)$

$\mathrm{Nr}^{\prime \prime}(1)=-2 \lambda^{+} \mathrm{m}_{1} \mathbf{I}_{\mathbf{0}} \mathbf{A}^{*}\left(\lambda^{+}\right) \sum_{\mathrm{i}=1}^{\mathrm{M}} \mathrm{p}_{\mathrm{i}} \mathrm{T}_{1}$ 


$$
\begin{aligned}
& \mathrm{D}^{\prime}(1)=\lambda^{-}\left(1-\mathrm{m}_{1}\left(1-\mathrm{A}^{*}\left(\lambda^{+}\right)\right)-\lambda^{+} \mathrm{m}_{1} \sum_{\mathrm{i}=1}^{\mathrm{M}} \mathrm{p}_{\mathrm{i}}\left(1-\mathrm{B}_{\mathrm{i}}^{*}\left(\lambda^{-}\right)\right)\left(\lambda^{-}\left(\phi_{\mathrm{i}, 1}^{(1)}+\beta_{\mathrm{i}, 1}^{(1)}\right)+\mathrm{T}_{1}\right)\right. \\
& \mathrm{D}^{\prime \prime}(1)=-\lambda^{-} \mathrm{m}_{2}\left(1-\mathrm{A}^{*}\left(\lambda^{+}\right)\right)-2 \lambda^{+} \mathrm{m}_{1} \mathrm{~T}_{1}+\mathrm{T}\left(1-\sum_{\mathrm{i}=1}^{\mathrm{M}} \mathrm{p}_{\mathrm{i}} \mathrm{B}_{\mathrm{i}}^{*}\left(\lambda^{-}\right)\right)+ \\
& 2 \lambda^{+} \mathrm{m}_{1}^{2}\left(1-\mathrm{A}^{*}\left(\lambda^{+}\right)\right) \sum_{i=1}^{\mathrm{M}} \mathrm{p}_{\mathrm{i}}\left(\mathrm{B}_{\mathrm{i}}^{*}\left(\lambda^{-}\right) \mathbf{T}_{\mathbf{1}}-\lambda^{-}\left(\mathbf{1}-\mathrm{B}_{\mathrm{i}}^{*}\left(\lambda^{-}\right)\right)\left(\phi_{\mathrm{i}, 1}^{(1)}+\beta_{\mathrm{i}, 1}^{(1)}\right)\right)+ \\
& -\sum_{\mathrm{i}=1}^{\mathrm{M}} \mathrm{p}_{\mathrm{i}}\left(-2 \lambda^{+}{ }^{2} \mathrm{~m}_{1}^{2}\left(\mu_{\mathrm{i}, 1} \mathbf{T}_{\mathbf{1}}{ }^{2}-\lambda^{-}\left(\left(\phi_{\mathrm{i}, 1}^{(1)}+\beta_{\mathrm{i}, 1}^{(1)}\right) \mu_{\mathrm{i}, 1} \mathbf{T}_{\mathbf{1}}-\left(1-\mathrm{B}_{\mathrm{i}}^{*}\left(\lambda^{-}\right)\right) \phi_{\mathrm{i}, 1}^{(1)} \beta_{\mathrm{i}, 1}^{(1)}\right)\right)\right. \\
& -\lambda^{-}{\underset{i=1}{M}}_{p_{i}}\left(1-B_{i}^{*}\left(\lambda^{-}\right)\right) \mathbf{T}_{2} \\
& \mathrm{~T}=-\alpha_{\mathrm{i}} \mathrm{T}_{2}-2 \alpha_{\mathrm{i}} \lambda^{+^{2}} \mathrm{~m}_{1}^{2}\left(\left(\phi_{\mathrm{i}, 1}^{(2)}+\beta_{\mathrm{i}, 1}^{(2)}\right)\left(\frac{1-\tau_{\mathrm{i}}}{\theta_{\mathrm{i}}}\right)+\phi_{\mathrm{i}, 1}^{(2)} \beta_{\mathrm{i}, 1}^{(2)}\right) \\
& -\frac{\alpha_{i} \lambda^{+}\left(1-\tau_{i}\right)}{\theta_{i}^{2}}\left[m_{2} \theta_{i}+2 \lambda^{+} m_{1}^{2}\right]-\lambda^{+} m_{2} \\
& \mathrm{~T}_{1}=\left[1+\alpha_{\mathrm{i}}\left(\phi_{\mathrm{i}, 1}^{(2)}+\beta_{\mathrm{i}, 1}^{(2)}+\frac{1-\tau_{\mathrm{i}}}{\theta_{\mathrm{i}}}\right)\right] \\
& \mathrm{T}_{2}=\lambda^{+2} \mathrm{~m}_{1}^{2}\left(\phi_{\mathrm{i}, 2}^{(2)}+\beta_{\mathrm{i}, 2}^{(2)}\right)+\lambda^{+} \mathrm{m}_{2}\left(\phi_{\mathrm{i}, 1}^{(2)}+\beta_{\mathrm{i}, 1}^{(2)}\right)
\end{aligned}
$$

The mean number of customers in the system $L_{s}$ is given by

$$
\mathrm{L}_{\mathrm{S}}=\mathrm{P}_{\mathrm{S}}^{\prime}(1)=\frac{\left[\mathrm{D}^{\prime}(1) \mathrm{Nr}_{1}^{\prime \prime}(1)-\mathrm{Nr}_{1}^{\prime}(1) \mathrm{D}^{\prime \prime}(1)\right]}{2 \mathrm{D}^{\prime}(1)^{2}}
$$

Let $\mathrm{Nr}_{1}(\mathrm{z})$ and $\mathrm{D}(\mathrm{z})$ represent the numerator and denominator of $\mathrm{P}_{\mathrm{q}}(\mathrm{z})$.

where,

$$
\begin{aligned}
& \mathrm{Nr}_{1}^{\prime}(1)=\mathrm{I}_{0} \lambda^{-} \mathrm{A}^{*}\left(\lambda^{+}\right) \\
& \mathrm{Nr}_{1}^{\prime \prime}(1)=-2 \lambda^{+} \mathrm{m}_{1} \mathrm{I}_{0} \lambda^{-} \mathrm{A}^{*}\left(\lambda^{+}\right) \sum_{\mathrm{i}=1}^{\mathrm{M}} \mathrm{p}_{\mathrm{i}} \mathrm{B}_{\mathrm{i}}^{*}\left[1+\alpha_{\mathrm{i}}\left(\phi_{\mathrm{i}, 1}^{(2)}+\beta_{\mathrm{i}, 1}^{(2)}+\frac{1-\tau_{\mathrm{i}}}{\theta_{\mathrm{i}}}\right)\right]
\end{aligned}
$$

\section{RELIABILITY INDICES}

Let $A(t)$ be the system availability at time $t$, that is the probability that the server is idle or working for a customer. Then under steady state condition,

\section{The availability of the server is given by}

$$
A=1-(H+F+Q)
$$$$
=1-\left[\lambda^{+} m_{1} \sum_{i=1}^{M} p_{i}\left(1-B_{i}^{*}\left(\lambda^{-}\right)\right)\left(\theta_{i}\left(\lambda^{-}\left(\phi_{i, 1}^{(1)}+\beta_{i, 1}^{(1)}\right)+\alpha_{i}\left(\phi_{i, 1}^{(2)}+\beta_{i, 1}^{(2)}\right)\right)+\alpha_{i}\left(1-\tau_{i}\right)\right) / \theta_{i} \lambda^{-}\right]
$$

The steady state failure frequency of the server is

$$
F=\lambda^{+} \mathrm{m}_{1} \sum_{\mathrm{i}=1}^{\mathrm{M}} \mathrm{p}_{\mathrm{i}}\left(1-\mathrm{B}_{\mathrm{i}}^{*}\left(\lambda^{-}\right)\right)\left(\lambda^{-}+\alpha_{\mathrm{i}}\right) / \lambda^{-}
$$

\section{STOCHASTIC DECOMPOSITION}

Theorem: The expected number of customers in the system $\mathrm{L}_{\mathrm{s}}$ can be expressed as sum of two independent random variables, one of which is the expected number of customers (L) in the unreliable batch arrival classical queueing system with positive and negative arrivals, multi-optional service, two types of breakdown and repair and the other is the expected number of customers in the orbit during idle time $\left(\mathrm{L}_{\mathrm{I}}\right)$.

\section{Proof:}

The probability generating function $\pi(\mathrm{z})$ of the system size of the classical unreliable batch arrival queue with multi-optional service, two types of breakdown and repair is given by

$$
\begin{aligned}
& \pi(\mathrm{z})= \lim ^{*} \mathrm{~A}^{*}\left(\lambda^{+}\right) \rightarrow 1 \\
&= \mathrm{P}_{1}(\mathrm{z}-1)\left(\sum_{\mathrm{i}=1}^{\mathrm{M}}(\mathrm{z})\right. \\
&\left.\mathrm{p}_{\mathrm{i}} \mathrm{B}_{\mathrm{i}}^{*}(\mathrm{~g}(\mathrm{z})) \mathrm{g}(\mathrm{z})+\lambda^{-} \sum_{\mathrm{i}=1}^{\mathrm{M}} \mathrm{p}_{\mathrm{i}}\left(1-\mathrm{B}_{\mathrm{i}}^{*}(\mathrm{~g}(\mathrm{z}))\right)\right) / \mathrm{D}_{1}(\mathrm{z})
\end{aligned}
$$

where

$$
\mathrm{I}_{1}=\frac{\lambda^{-}-\lambda^{+} \mathrm{m}_{1} \sum_{\mathrm{i}=1}^{\mathrm{M}} \mathrm{p}_{\mathrm{i}}\left(1-\mathrm{B}_{\mathrm{i}}^{*}\left(\lambda^{-}\right)\right)\left(\lambda^{-}\left(\phi_{\mathrm{i}, 1}^{(1)}+\beta_{\mathrm{i}, 1}^{(1)}\right)+\mathrm{T}_{1}\right)}{\lambda^{-}}
$$

The probability generating function $\chi(\mathrm{z})$ of the number of customers in the orbit when the system is idle is given by

$$
\begin{aligned}
& \chi(\mathrm{z})=\frac{\mathrm{I}_{0}+\mathrm{I}(\mathrm{z})}{\mathrm{I}_{0}+\mathrm{I}(1)} \\
& =\frac{\left(\mathrm{zg}(\mathrm{z})-\sum_{\mathrm{i}=1}^{\mathrm{M}} \mathrm{p}_{\mathrm{i}}\left[\mathrm{g}(\mathrm{z}) \mathrm{B}_{\mathrm{i}}^{*}(\mathrm{~g}(\mathrm{z}))+\lambda^{-}\left(1-\mathrm{B}_{\mathrm{i}}^{*}(\mathrm{~g}(\mathrm{z})) \mathrm{S}_{\mathrm{i}}^{(1)^{*}}(\mathrm{~h}(\mathrm{z})) \mathrm{R}_{\mathrm{i}}^{(1)^{*}}(\mathrm{~h}(\mathrm{z}))\right]\right)\right)}{\left.\mathrm{D}(\mathrm{z}) \lambda^{-}-\lambda^{+} \mathrm{m}_{1} \sum_{\mathrm{i}=1}^{\mathrm{M}} \mathrm{p}_{\mathrm{i}}\left(1-\mathrm{B}_{\mathrm{i}}^{*}\left(\lambda^{-}\right)\right)\left(\lambda^{-}\left(\varphi_{\mathrm{i}, 1}^{(1)}+\beta_{\mathrm{i}, 1}^{(1)}\right)+\mathrm{T}_{1}\right)\right)}
\end{aligned}
$$

From equations (58), (68) and (69) we see that $\mathrm{P}_{\mathrm{S}}(\mathrm{z})=\pi(\mathrm{z}) \bullet \chi(\mathrm{z})$ and hence $\mathrm{L}_{\mathrm{s}}=\mathrm{L}+\mathrm{L}_{\mathrm{I}}$.

\section{REFERENCES}

[1] Aissani, A. (1988). On the M/G/1 Queueing system with Repeated Orders and Unreliable Server, Journal of Technology, Vol.6, pp.98-123.

[2] Choudhury, G. and Ke, J.C. (2012). A Batch Arrival Retrial Queue with General Retrial Times Under Bernoulli Vacation Schedule for Unreliable Server and Delayed Repair, Applied Mathematical Modelling, Vo.36, pp.255-269.

[3] Falin, G. I. (1990). A Survey of Retrial Queues, Queueing Systems, Vol.7, pp.127-169.

[4] Gelenbe, E.(1989). Random Neural Networks with Negative and Positive Signals and Product Form Solution. Neural Computation, 1,502- 510.

[5] Kirupa, K. and Udaya Chandrika, K. (2014). Batch arrival Retrial G-queue and an unreliable server with delayed repair, International Journal of Innovative Research in Science, Engineering and Technology, Vol. 3, Issue 5, May 2014.

[6] Kulkari, V.G. and Choi, B.D. (1990). Retrial Queues with Server Subject to Breakdowns and Repairs, Queueing Systems, Vol.7,No. 2, pp.191-208.

[7] Peng, Y., Liu, Z. and Wu, J. (2013). An M/G/1 Retrial GQueue with Preemptive Resume Priority and Collisions Subject to the Server Breakdowns and Delayed Repairs, Journal of Applied Mathematical Computations, doi.10.1007/s12190-013-0688-7.

[8] Prakash Rani, K., Srinivasan, A. and Udaya Chandrika, K. (2009). Impatient Customers Bulk Arrival Retrial Queue with Server Breakdown and Delayed Repair, Advances in Applied Research, Vol.1, No.1,pp.60-68.

[9] Wang, J. and Zhang, P. (2009). A Single-Server Discrete-Time Retrial G-queue with Server Breakdowns and Repairs, Acta Mathematicae Applicatae Sinica, Vol.25, pp.675-684.

[10] Yang, T. and Templeton, J.G.C. (1987). A Survey on Retrial Queues, Queueing Systems, Vol.2, No.3, pp.201233 